\title{
Vertebral body replacement with an expandable cage for reconstruction after spinal tumor resection
}

\author{
Issada Thongtrangan, M.D., Raju S. V. Balabhadra, M.D., Hoang Le, M.D., \\ Jon PaRK, M.D., F.R.C.S.C., AND DANIEL H. KIM, M.D. \\ Department of Neurosurgery, Stanford University Medical Center, Stanford, California
}

\begin{abstract}
Object. The authors report their clinical experience with expandable cages used to stabilize the spine after vertebrectomy. The objectives of surgical treatment for spine tumors include a decrease in pain, decompression of the neural elements, mechanical stabilization of the spine, and wide resection to gain local control of certain primary tumors. Most of the lesions occur in the anterior column or vertebral body (VB). Anterior column defects following resection of VBs require surgical restoration of anterior column support. Recently, various expandable cages have been developed and used clinically for VB replacement (VBR).

Methods. Between January 2001 and June 2003, the authors treated 15 patients who presented with primary spinal tumors and metastatic lesions from remote sites. All patients underwent vertebrectomy, VBR with an expandable cage, and anterior instrumentation with or without posterior instrumentation, depending on the stability of the involved segment. The correction of kyphotic angle was achieved at an average of $20^{\circ}$. Pain scores according to the visual analog scale decreased from 8.4 to 5.2 at the last follow-up review. Patients whose Frankel neurological grade was below D attained at least a one-grade improvement after surgery. All patients achieved immediate stability postsurgery and there were no significant complications related to the expandable cage.

Conclusions. The advantage of the expandable cage is that it is easy to use because it permits optimal fit and correction of the deformity by in vivo expansion of the device. These results are promising, but long-term follow up is required.
\end{abstract}

KEY WORDS $\bullet \quad$ spinal tumor $\bullet$ vertebrectomy $\bullet$ spinal fusion $\bullet$
spinal instrumentation $\bullet$ vertebral body replacement $\bullet$ expandable cage

The spinal column is the most common site of bone metastasis. ${ }^{4}$ In patients who are asymptomatic, the thoracic spine is involved in $70 \%$ of cases, whereas the cervical and lumbar regions are affected in 10 and 20\%, respectively. ${ }^{8}$ Metastatic carcinoma from the lungs, prostate, breasts, kidneys, thyroid, and gastrointestinal tract account for the majority of spinal column tumors. Other lesions that commonly metastasize to the spine include myeloma and lymphoma. Certain primary tumors (chordoma, osteoblastoma) tend to occur most frequently in the spinal column; however, they represent less than $2 \%$ of all spine tumors. ${ }^{4}$

The objectives of surgical treatment for spine tumors include a decrease in pain, decompression of the neural elements, mechanical stabilization of the spine, and wide resection to gain local control of certain primary tumors. Most of the lesions occur in the body of the vertebra. Anterior column defects caused by resection of VBs require surgical restoration of anterior column support. In recent years, several artificial materials and implants have

Abbreviations used in this paper: $\mathrm{CT}=$ computerized tomography; VAS = visual analog scale; VB = vertebral body; VBR = VB replacement. been developed to replace the VB, and bone cement is still widely accepted for this purpose. ${ }^{10}$ Titanium cages, ${ }^{1}$ ceramic, ${ }^{11,14}$ ceramic/glass, ${ }^{13}$ and carbon fiber spacers; ${ }^{3}$ and different special implants ${ }^{17}$ are also used clinically.

Recently, various expandable cages have been developed and used clinically for VBR, although there is a paucity of literature regarding the outcomes and results of using synthetic vertebral spacers and expandable cages. The advantage of the VBR expandable cage is that it is easy to place because it permits an optimal, tight fit and correction of the deformity by in vivo expansion of the device. Our primary goal in this paper is to report our clinical experience in the use of expandable VBR cages in patients with tumors.

\section{CLINICAL MATERIAL AND METHODS Patient Population}

Using a local database and chart reviews, we retrospectively identified 15 consecutive patients who underwent expandable cage reconstruction of the anterior spinal column after single- or multilevel vertebrectomy between C-7 and L-3 for spinal tumors between January 2001 and June 2003. Neuroimaging and functional outcome evaluations were performed in all 15 patients at a mean follow- 
up period of 12.6 months (range 2-22 months). The functional outcomes were analyzed using the VAS, ${ }^{20}$ and the neurological performance scale proposed by Frankel, et al., ${ }^{6}$ and perioperative complications were documented. There were seven women and eight men whose mean age was 55.7 years (range 30-79 years).

The clinical diagnosis and demographic data are shown in Table 1. On presentation, three patients were classified as neurologically normal (Frankel Grade E), six as Frankel Grade D, five as Frankel Grade C, and one as Frankel Grade B. The six patients who were classified as Frankel Grades B and C had bowel or bladder dysfunction on admission. The reconstructions were performed in the thoracic spine (T2-12) in 10 patients, in the lumbar spine (L1-5) in four, and in the cervical spine in one. Seven of the patients had lesions involving the thoracolumbar junction (T11-L2). Eleven patients underwent a one-level vertebrectomy, whereas two- and three-level vertebrectomies were performed in three patients and in one patient, respectively.

Radiographic images will be used to determine the degree of correction of the kyphotic angle between the pre- and postoperative phase, stability of the VBR cage, and complications related to the cage. Spinal fusion was not our primary goal in these patients, because most of them received adjuvant radiotherapy and chemotherapy to control the underlying disease.

Descriptive statistics (means \pm standard deviations) were obtained for quantitative variables, and percentages were calculated for categorical variables of interest.

\section{Biomechanical Properties of VBR}

Biomechanical studies were performed to determine potential failure modes and their associated causes, as well as to meet the American Society for Testing and Materials standard. The VBR cage is most stable mechanically when it is not distracted, because the compression load is distributed over the cross-sectional area of both the center piece and the end piece. In addition, because the end pieces are linked to the center piece by threads, the more threads engaged (a less distracted state), the stronger the assembly. Figure 1 depicts the VBR expandable cage.

Compression Testing. Using a biomechanical model corroborated by measurements of the myoelectric activities in the trunk and intradiscal pressure, Schultz, et al., ${ }^{21}$ predicted mean compression loads on the lumbar spine of up to $2400 \mathrm{~N}$ with the individual in a standing position holding two 4-kg weights in outstretched hands and with the trunk flexed at $30^{\circ}$. Thus, the results of the VBR testing (12,720-N static failure load; 5 million cycles at 4-kN dynamic loading) clearly demonstrate that this device exceeds the minimum strength criteria in the published literature.

Shear Testing. The failure loads were comparable and failure modes were the same for both shear testing and compression testing. For static shear testing the mean failure load was $16,459 \mathrm{~N}$ and for static compression testing the mean failure load was $12,720 \mathrm{~N}$. For dynamic testing the 5-million-cycle runout load in both shear and compression testing was at least $4000 \mathrm{~N}$. The failure mode in

TABLE 1

Demographic data in patients who underwent VBR with an expandable cage*

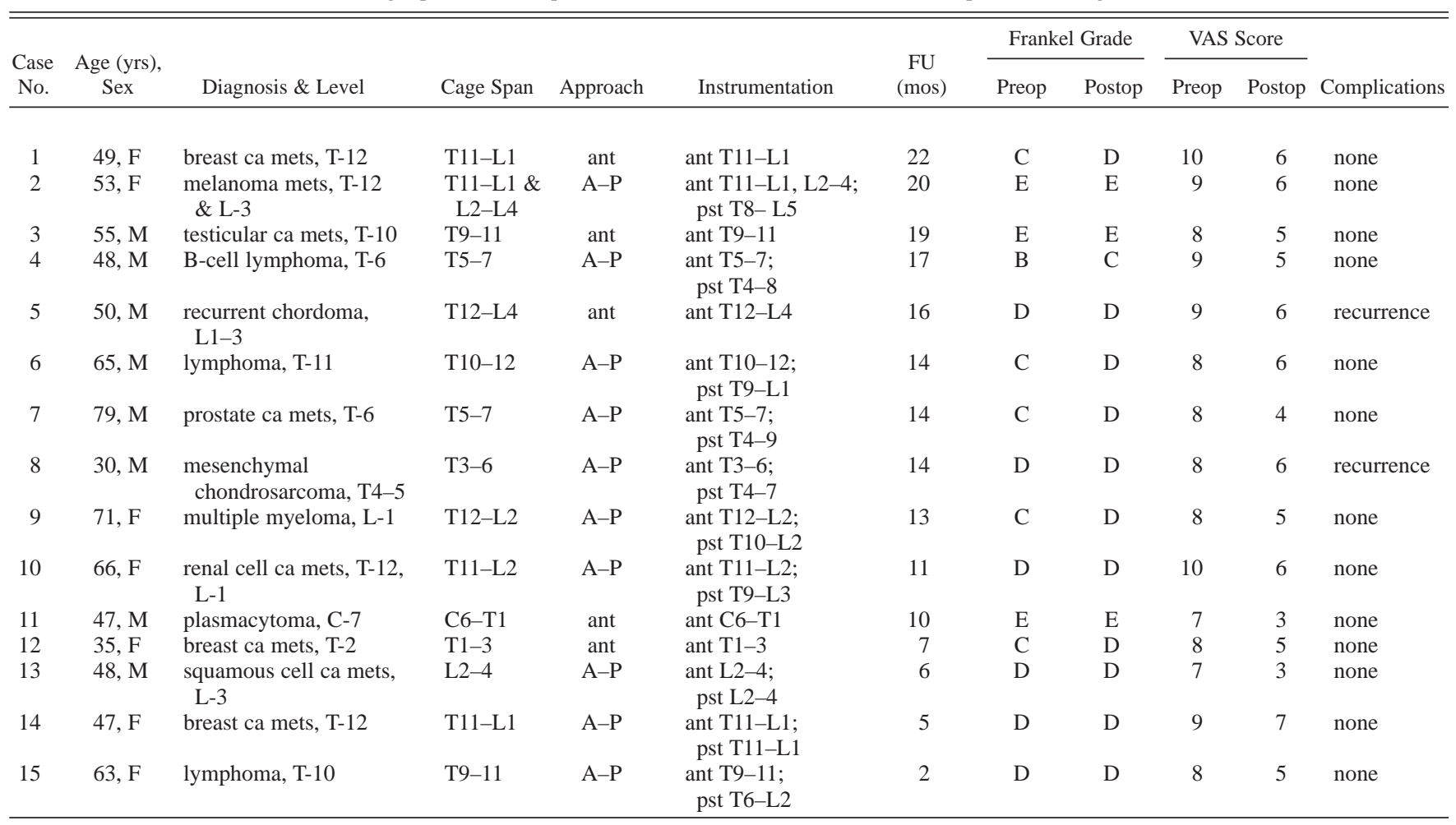

$*$ Ant $=$ anterior; $\mathrm{A}-\mathrm{P}=$ anterior-posterior; $\mathrm{ca}=$ carcinoma FU = follow up mets = metastasis $;$ pst = posterior . 


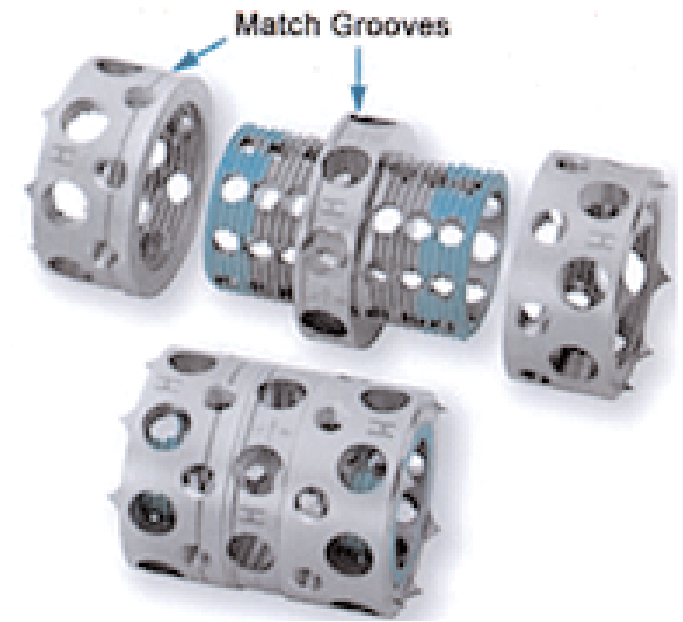

Fig. 1. Photograph showing the expandable cage device consisting of one central core and two end pieces, with small holes made around the device for the purpose of bone fusion. To assemble, the match grooves of the central core and the end piece need to be aligned on the same side. The spikes at both ends increased the stability of the device at the bone-implant interface; however, it is not designed to be used as a standalone device. Both ends can be changed from flat $\left(0^{\circ}\right)$ to $5^{\circ}$ or $8^{\circ}$.

each of the various types of testing was shearing of the VBR threads. Thus, VBR devices with angled end pieces did not endure significant shear forces under compressive loading, but rather exhibited mechanical characteristics very similar to the VBR constructs without angled end pieces subjected to compression loads. In addition, the results of the dynamic shear testing (5 million cycles at 4 $\mathrm{kN}$ ) demonstrated that the VBR devices with angled end pieces exceed the offset deformable barrier's minimum requirement of the American Society for Testing and Materials of 5 million cycles at $3 \mathrm{kN}$.

Torsional Testing. The results show that the maximum runout load to 5 million cycles without failure was 4.5 $\mathrm{Nm}$. Failure of the constructs occurred in the upper region of the center piece, right above the central flange. No loosening of the constructs was observed after testing. The mean ultimate static torsional strength was $9.22 \mathrm{Nm}$. Thus, the mean ultimate static torsional strength of 9.22 $\mathrm{Nm}$ and the torsional fatigue strength of $4.5 \mathrm{Nm}$ at 5 million cycles recorded for the VBR device demonstrate that it can withstand the torsional loads to which it would be subjected in vivo.

Expulsion Testing. A standard of deviation of $6.69 \mathrm{~N}$ with a mean ultimate load of $406.32 \mathrm{~N}$ was found for the constructs tested with a preload of $500 \mathrm{~N}$. The mode of failure seen during testing was the failure of the polyurethane foam due to gouging of the metal teeth of the endplates in the foam blocks. No failure or deformation of the VBR construct was apparent or detected in any of the tests.

Conclusions. From the biomechanical standpoint, the VBR construct's mechanical properties are capable of withstanding the loads, and the device appears to be effective for its intended use of replacing VBs after vertebrectomy and providing stabilization of the spine by restoring and/or maintaining vertebral height.

\section{Surgical Procedure}

All patients underwent VB resection and decompression of the spinal canal, followed by anterior column reconstruction with an expandable cage. The disc and cartilage were removed while preserving the bone endplate, although the process of cage insertion routinely resulted in some disruption of the endplate from the edges of the cage. The appropriate implant size was determined using a caliper to measure the length of the corpectomy to be bridged, and the measuring template was used next to match the distance of the corpectomy with the appropriate VBR implant.

The matching VBR end pieces were attached to the corresponding central core after sizing. Angled end pieces that matched the anatomy of the vertebral endplates were selected, and these end pieces could be used in combination to provide $0,5,8,10,13$, and $16^{\circ}$ of angulation based on the severity of deformity.

Graft material could be used to fill the VBR device; we used autograft, allograft, and calcium phosphate in our series. Once the construct is distracted to its final length, additional graft material could be added to fill the end pieces completely. With angled end pieces implantation was critical; otherwise the device could rotate and lead to failure of correction. The surgeon had to control the cage's position during its implantation and expansion. The cage can be expanded either with an expansion instrument or wrench. The implant position was confirmed after the VBR assembly had been appropriately expanded. If repositioning was required, the expansion instrument was turned in the opposite direction to close the VBR assembly, which was repositioned and then reexpanded. Once correct positioning of the VBR was confirmed, the end pieces were secured to the central core with two set screws in each end piece by using a screwdriver. If desired, additional bone graft or bone substitute could be placed around the implant. Because the cage is not designed to be a standalone device, an anterior plate or rod construct was placed in all cases. If the posterior column integrity was compromised, or if more extensive vertebral column reconstruction was needed, then same-day combined anterior-posterior decompression and stabilization procedures were performed. In our series, 10 patients (66.7\%) underwent a same-day combined anterior-posterior approach, whereas five $(33.3 \%)$ underwent an anterior approach only.

\section{Radiographic Outcomes}

\section{RESULTS}

The correction of the kyphotic angle achieved postoperatively averaged $20^{\circ}$. There were no significant complications related to cage placement. At the end of the follow-up period, there were no instances of cage or hardware failures. All patients achieved immediate stability after the surgeries. Fusion was not our primary goal in these patients because most of them required adjuvant chemotherapy and radiotherapy thereafter. One of the patients in our series had undergone CT scanning postoperatively, however, and the CT scan revealed good alignment of the expandable cage, proper placement of the hardware, and that the VBR cage appeared to fit tightly to the host bone (Fig. 2). 


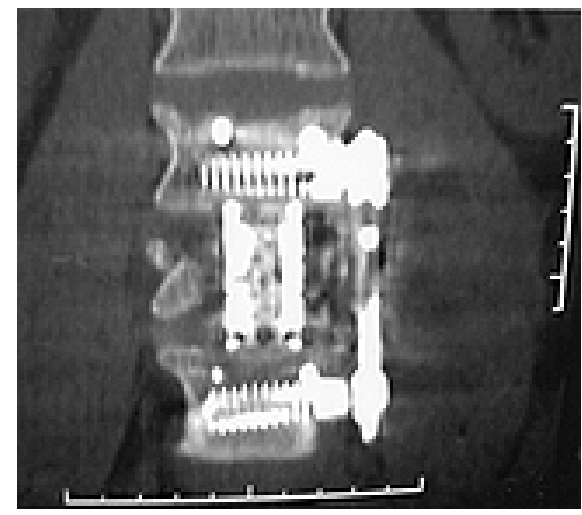

Fig. 2. Case 13. A coronal reformatted CT scan demonstrating good alignment of the expandable cage after vertebrectomy.

\section{Functional Outcomes}

The VAS (Pain Scale). At the end of the follow-up period, patients exhibited significant improvements in their mean pain scale or VAS scores (the VAS scores range from 0 to 10 , with 10 being the most painful). Their pain levels decreased from 8.4 preoperatively to 5.2 at the final follow-up visit.

The Frankel Neurological Performance Scale. Patients who were classified as Frankel Grade C or below preoperatively improved by at least one neurological grade postoperatively. Those who began at Frankel Grades D or E remained the same after the surgery. Recurrence of the tumor was found in two patients at the final follow-up review.

Perioperative Complications. An anterior approach was performed in five patients, whereas a combined anterior-posterior approach was performed in 10 patients. No perioperative (approach-related) complications were noted in our study. There was no incidence of disease transmission, superficial or deep wound infections, or neurological deterioration. At a mean follow-up time of 12.6 months, there were also no complications related to the expandable cages. There were, however, two cases of recurrent disease: the patient in Case 5, who had a recurrent chordoma, and the one in Case 8, who had a mesenchymal chondrosarcoma.

\section{ILLUSTRATIVE CASE STUDY}

\section{Case 1}

This 49-year-old woman presented with severe back pain, bilateral lower-extremity weakness, and urinary incontinence. Neurological examination revealed her Frankel grade as $\mathrm{C}$ at her initial presentation. She was known to have had underlying breast cancer for a few years. Magnetic resonance images and reformatted CT scans of the thoracolumbar spine revealed severe destruction of the T-12 VB by tumor, and compression of the spinal canal (Fig. 3). Via a left anterolateral approach, the tumor and the T-12 VB were resected, and the spinal cord was completely decompressed. The expandable cage was inserted, spanning T-11 to L-1, and anterior plating (MACS-TL; Aesculap, Tuttlingen, Germany) was placed from T-11 to

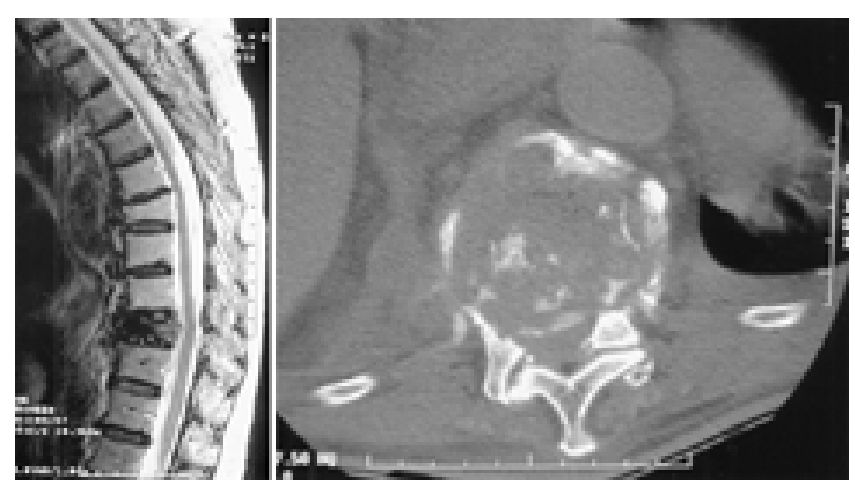

Fig. 3. Case 1. Neuroimages obtained in a 49-year-old woman with metastatic breast cancer. Left: Sagittal $\mathrm{T}_{2}$-weighted magnetic resonance image revealing severe destruction of the VB at T-12 with significant kyphotic deformity and canal compromise. Right: Axial CT scan revealing anterior element destruction at T-12 with spinal canal compromise.

L-1. We did not perform supplemental posterior fixation because the posterior vertebral column was intact and VBR with the anterior construct was biomechanically strong enough in this patient. Postoperative $\mathrm{x}$-ray films demonstrated correction of the deformity and confirmed that the expandable cage spanned from T-11 to L-1 (Fig. $4)$. The patient's pain was significantly reduced immediately after the surgery, and her neurological grade improved by one increment, to a Frankel Grade D, after the surgery.

\section{DISCUSSION}

In recent years, several artificial materials and implants have been developed to replace the VB, although bone cement is still widely accepted for this purpose.$^{10}$ Other options are various kinds of synthetic cages made from carbon fiber, titanium fiber mesh, or expandable titanium cages, and they have become popular substitutes for structural bone grafts. Implantation of synthetic cages into the anterior column seems to offer immediately effective segmental stability, correction of the sagittal plane deformity, and restoration of the anterior vertebral support from a biomechanical standpoint. These anterior interbody cages provide a satisfactory axial load-bearing capacity, and morcellized autograft can be used to fill the inside of the cage. Recently, various types of cages have been used clinically and are classified as carbon fiber, mesh, and titanium metal, based on the material's characteristics.

Carbon fiber cages have the advantage of radiolucency; therefore, they offer better opportunities for postoperative fusion assessment. Unfortunately, there are disadvantages to using carbon fiber implants, including inflammatory responses with possible foreign body reactions and brittleness resulting in breakage, as well as composite material failure. ${ }^{24}$

Vertical metallic mesh cages are also a viable option for anterior column support with minimal complications. The titanium mesh cage allows anchoring by bone, because its end tip has spikes. Unlike bone grafts, both ends of a titanium mesh cage have sharp edges that allow it to be anchored into the adjacent VBs, which results in torsional 


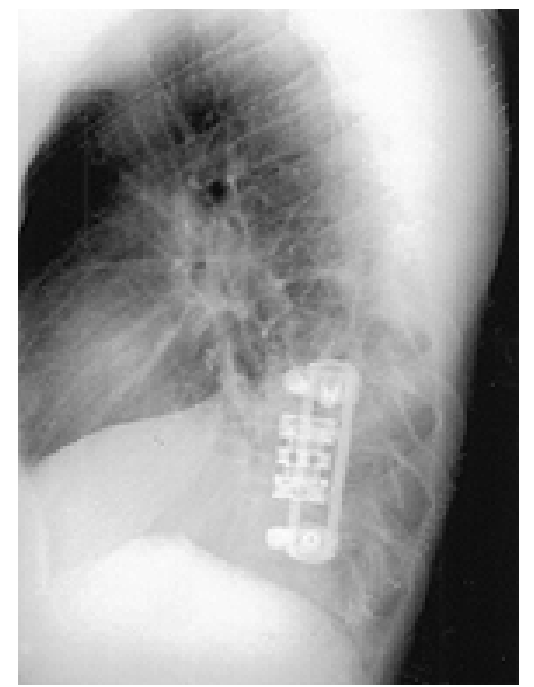

Fig. 4. Postoperative lateral x-ray image revealing correction of the preoperative kyphotic deformity after vertebrectomy and expandable cage placement from T-11 to L-1 with anterior instrumentation.

stability. The vertical placement of the mesh cage allows optimal room for autogenous grafting and for contact of the graft with the vertebral endplates. The potential for radiographic assessment of mesh cages is not as good as in the carbon fiber implants with their radiolucency. Subsidence of the mesh cage into adjacent VBs and lateral displacement are the most common complications when adjacent VBs were of optimal quality. ${ }^{23}$

One of the recent advancements in VBR procedures is the variety of forms of the expandable titanium vertebral cage. These cages are highly versatile and easily expandable and can be used to reconstruct the vertebral column defect and to correct the sagittal alignment of the spine. They can facilitate distraction across the resected vertebral defect for correction of the deformity, allow immediate load bearing after corpectomy, and provide a satisfying long-term functional result. The risk of disease transmission associated with allografts is avoided as well. Even though fusion for long segmental vertebral defects is needed, immediately stability can be maintained without any settling of the graft.

One potential complication associated with the use of titanium mesh cages as anterior column supports is subsidence into the VBs. On the other hand, very minimal subsidence occurs with expandable cages because of their broader surface and duller edges at each end. After successful VB reconstruction with the expandable cage, the anterior fixation device or the posterior instrumentation must be added, because this cage provides only minimal stability in all directions of load, particularly in the torsional load of the segment. The major disadvantages of these expandable cages are that they prevent adequate radiographic assessment of fusion and offer a smaller surface as a fusion bed than titanium mesh. Nevertheless, fusion is not the primary goal for patients with tumors.

There were no significant intra- or postoperative complications. At a mean follow-up time of 12.6 months, there were also no complications related to the expandable cages. There were, however, two instances of recurrent disease: the patient in Case 5, who had a recurrent chordoma, and the one in Case 8, who had a mesenchymal chondrosarcoma. Given the limitations of this study, however, the expandable cage appears to be safe and effective. The results are promising for providing immediate stability and reducing pain significantly.

We believe that the use of expandable cages in spinal tumor reconstruction is worthwhile because of the following reasons: 1) easier placement after vertebrectomy; 2) ability to fit tightly after expansion of the cage; and 3) ability to correct the deformity.

\section{Significance of the Anterior Approach}

Most of the axial load of the spine is carried by the anterior vertebral column. Therefore, anterior column reconstruction is the most important component in the restoration of normal spinal biomechanics. The ideal anterior column support must provide immediate stability and permit successful bone fusion. Several authors have studied the use of short-segment posterior pedicular fixation in patients with trauma and found high rates of instrumentation failure, even though transpedicular bone grafting has been performed. ${ }^{2,5,15,16}$ Most of the failures occurred because of cantilever bending forces acting on the screws in the absence of a competent anterior column. On the other hand, anterior constructs have some obvious advantages and offer a safe short-segment fixation for stability. Several biomechanical studies have revealed that in the thoracolumbar area, anterior constructs were more rigid in axial compression, torsion, and flexion loading than posterior constructs. $^{9,22}$

Posterior instrumentation without anterior reconstruction has been used to reduce deformity and stabilize the spine in cases of incompetent anterior column defects. Several authors, however, have reported failure of the posterior constructs to maintain stability in cases of compromised anterior column. ${ }^{5,15}$ There have been reports of failure of anterior constructs consisting of tricortical strut bone graft and anterior instrumentation in thoracolumbar fractures because the graft could not withstand the axial compression load. ${ }^{7,25}$ Therefore, a combined or staged posterior fixation was often required to supplement anterior constructs. Oda, et al., ${ }^{18}$ demonstrated that a combined anterior-posterior fixation was biomechanically superior to either an anterior construct alone or a posterior construct alone in thoracolumbar total vertebrectomy. Kandziora, et al., ${ }^{12}$ in a biomechanical study of expandable cages after cervical corpectomy, concluded that anterior plating increased biomechanical stability adequately and that additional posterior stabilization should only be considered in cases of severe rotational instability of the cervical spine. In most of the patients in our series, lesions occurred in the thoracic region and at the thoracolumbar junction; therefore, we believed that a combined construct was the most stable after total vertebrectomy.

Complications may result from the surgical approach and from the placement of spinal instrumentation. The anterior approach may pose a risk of great-vessel injury as well as internal organ injury because of unfamiliar anatomical landmarks or excessive traction. A retroperitoneal approach also requires careful protection of the ureter, 
lumbosacral plexus, and sympathetic chain. In a recent retrospective study, Oskouian and Johnson ${ }^{19}$ reported a $5.8 \%$ incidence (12 of 207 patients) of vascular complications in patients who underwent anterior approaches in the thoracic and lumbar spine, but without serious consequences. In our series we observed no vascular and internal organ complications; however, it is our opinion that the benefits of the anterior approach are outweighed by the risks.

\section{CONCLUSIONS}

Expandable cages allow restoration and preservation of height, lordosis, and alignment after anterior tumor resection from the cervical spine to the lumbar spine, especially at the thoracolumbar junction. In our experience there were no instrumentation failures. This device provides an additional means of achieving reduction of kyphotic deformity and stabilization after tumor resection. In our series the expandable cage appears to be effective after vertebrectomy; however, it is not designed for use as a standalone device. Anterior instrumentation with or without additional posterior instrumentation is required. The advantage of the expandable cage is that it is easy to use because it permits optimal fit and correction of the deformity because of in vivo expansion of the cage. Our early results are promising, but long-term studies are needed.

\section{References}

1. Akamaru T, Kawahara N, Tsuchiya H, et al: Healing of autologous bone in a titanium mesh cage used in anterior column reconstruction after total spondylectomy. Spine 27:E329-E333, 2002

2. Alanay A, Acaroglu E, Yazici M, et al: Short-segment pedicle instrumentation of thoracolumbar burst fractures: does transpedicular intracorporeal grafting prevent early failure? Spine 26: 213-217, 2001

3. Brantigan JW, McAfee PC, Cunningham BW, et al: Interbody lumbar fusion using a carbon fiber cage implant versus allograft bone. An investigational study in the Spanish goat. Spine 19: 1436-1444, 1994

4. Cohen DB: Tumors of the spine, in Koval KJ (ed): Orthopaedic Knowledge Update: 7. Rosemont, IL: American Academy of Orthopaedic Surgeons Publishers, 2002, pp 673-687

5. Ebelke DK, Asher MA, Neff JR, et al: Survivorship analysis of VSP spine instrumentation in the treatment of thoracolumbar and lumbar burst fractures. Spine 16 (Suppl 8):S428-S432, 1991

6. Frankel HL, Hancock DO, Hyslop G, et al: The value of postural reduction in the initial management of closed injuries of the spine with paraplegia and tetraplegia. I. Paraplegia 7: 179-192, 1969

7. Ghanayem AJ, Zdeblick TA: Anterior instrumentation in the management of thoracolumbar burst fractures. Clin Orthop 335:89-100, 1997

8. Gokasalan ZL, York JE, Walsh GL, et al: Transthoracic vertebrectomy for metastatic spinal tumors. J Neurosurg 89: 599-609, 1998
9. Gurr KR, McAfee PC, Shih CM: Biomechanical analysis of anterior and posterior instrumentation systems after corpectomy. A calf-spine model. J Bone Joint Surg Am 70:1182-1191, 1988

10. Harrington KD: Anterior decompression and stabilization of the spine as a treatment for vertebral collapse and spinal cord compression from metastatic malignancy. Clin Orthop 233: 177-197, 1988

11. Hosono N, Yonenobu K, Fuji T, et al: Orthopaedic management of spinal metastases. Clin Orthop 312:148-59, 1995

12. Kandziora F, Pflugmacher R, Schaefer J, et al: Biomechanical comparison of expandable cages for vertebral body replacement in the cervical spine. J Neurosurg (Spine 1) 99:91-97, 2003

13. Kaneda K, Takeda N: Reconstruction with a ceramic vertebral prosthesis and Kaneda device following subtotal or total vertebrectomy in metastatic thoracic and lumbar spine, in Bridwell KH, DeWald RL (eds): The textbook of spinal surgery, ed 2. Philadelphia: Lippincott-Raven, 1997, Vol 2, pp 2071-2087

14. Matsui H, Tatezaki S, Tsuji H: Ceramic vertebral body replacement for metastatic spine tumors. J Spinal Disord 7:248-254, 1994

15. McCormack T, Karaikovic E, Gaines RW: The load sharing classification of spine fractures. Spine 19:1741-1744, 1994

16. McLain RF, Sparling E, Benson DR: Early failure of short-segment pedicle instrumentation for thoracolumbar fractures. A preliminary report. J Bone Joint Surg Am 75:162-167, 1993

17. Miller DJ, Lang FF, Walsh GL, et al: Coaxial double lumen methylmethacrylate reconstruction in the anterior cervical and upper thoracic spine after tumor resection. J Neurosurg (Spine 2) 92:181-190, 2000

18. Oda I, Cunningham BW, Abumi K, et al: The stability of reconstruction methods after thoracolumbar total spondylectomy. An in vitro investigation. Spine 24:1634-1638, 1999

19. Oskouian RJ Jr, Johnson JP: Vascular complications in anterior thoracolumbar spinal reconstruction. J Neurosurg (Spine 1) 96: $1-5,2002$

20. Scott J, Huskisson EC: Graphic representation of pain. Pain 2: 175-184, 1976

21. Schultz A, Andersson G, Orthengren R, et al: Loads on the lumbar spine. Validation of a biomechanical analysis by measurements of intradiscal pressures and myoelectric signals. J Bone Joint Surg Am 64:713-720, 1982

22. Shono Y, McAfee PC, Cunningham BW: Experimental study of thoracolumbar burst fractures. A radiographic and biomechanical analysis of anterior and posterior instrumentation systems. Spine 19:1711-1722, 1994

23. Theodore N, Vishteh AG, Baskin JJ, et al: Titanium mesh cage interbody fusion in the thoracolumbar spine. Tech Neurosurg 7:119-126, 2001

24. Tullberg T: Failure of a carbon fiber implant. A case report. Spine 23:1804-1806, 1998

25. Zdeblick TA, Shirado O, McAfee PC, et al: Anterior spinal fixation after lumbar corpectomy. A study in dogs. J Bone Joint Surg Am 73:527-534, 1991

Manuscript received September 16, 2003.

Address reprint requests to: Daniel H. Kim, M.D., Department of Neurosurgery, Stanford University Medical Center, Room R-201, Edwards Building, 300 Pasteur Drive, Stanford, California 943055327. email: neurokim@stanford.edu. 